\title{
AVALIAÇÃO DA DOSAGEM E TEMPO DE SEDIMENTAÇÃO NO TRATAMENTO DE ÁGUA SUPERFICIAL DE BAIXA TURBIDEZ COM COAGULANTE A BASE DE MORINGA OLEIFERA LAM.
}

\author{
A. T. A. BAPTISTA ${ }^{1}$, P. H. F. CARDINES ${ }^{1}$, M. O. SILVA ${ }^{2}$, R. BERGAMASCO $^{2}$, R. G. \\ GOMES $^{1}$ e A. M. S. VIEIRA ${ }^{1}$ \\ ${ }^{1}$ Universidade Estadual de Maringá, Departamento de Engenharia de Alimentos \\ ${ }^{2}$ Universidade Estadual de Maringá, Departamento de Engenharia Química \\ E-mail para contato: @gmail.com
}

RESUMO - Para um controle de qualidade da água é necessário o monitoramento de diversos indicadores. Muitos coagulantes químicos são amplamente utilizados nos processos de tratamento de água, porém geram lodos não biodegradáveis e podem ter ligação com algumas doenças, como o mal de Alzheimer o qual está relacionado ao alumínio residual presente nas águas. Desta forma tem aumentado o interesse pelo uso de coagulantes naturais como, por exemplo, a semente de Moringa oleifera Lam a qual contêm agentes ativos com excelente atividade e propriedades coagulantes. Este trabalho teve como objetivo estabelecer a melhor dosagem de coagulante de Moringa a ser utilizada e avaliar os diferentes tempos de sedimentação após a coagulação/floculação a fim de se otimizar o processo de tratamento de água superficial. Com os resultados obtidos foi possível encontrar a dosagem ótima de coagulante de 14,0 mg/L e o melhor tempo de sedimentação no processo de tratamento de água de 30 minutos. $\mathrm{O}$ coagulante de Moringa demonstrou ser uma boa alternativa para o tratamento de água bruta na redução da turbidez e cor, porém ainda se faz necessário aprimoramento da técnica para que sejam atingidos parâmetros de qualidade estabelecidos pela legislação.

\section{INTRODUÇÃO}

Para um controle da qualidade da água é necessário o monitoramento de diversos indicadores. A preocupação com a contaminação de ambientes aquáticos aumenta principalmente quando a água é usada para o consumo humano. Muitos coagulantes químicos são amplamente utilizados nos processos de tratamento de água com base nas suas características de coagulação e floculação, porém geram lodos não biodegradáveis e podem ter ligação com algumas doenças, como o mal de Alzheimer o qual está relacionado ao alumínio residual presente nas águas (Okuda et al., 2001). Tendo em vista esta problemática, tem-se aumentado o interesse pelo uso de polímeros naturais no tratamento de águas superficiais para a produção de água potável devido às grandes vantagens em relação aos agentes coagulantes químicos (Kawamura, 1991). Um destes 
coagulantes naturais é a semente da árvore tropical Moringa oleifera Lam a qual contém agentes ativos com excelente atividade e propriedades coagulantes.

A Moringa oleifera Lam é uma das espécies originária do nordeste indiano, cresce em regiões desde as subtropicais secas e úmidas, até tropicais secas e florestas úmidas (Gallão, 2006). A eficiência da semente de Moringa como coagulante natural para o tratamento de águas superficiais e residuárias já foi comprovada por vários autores (Madrona et al., 2010; Vieira, et al., 2010; Nishi et al., 2011). Algumas pesquisas reportam que a ação clarificante da Moringa está relacionada à presença de uma proteína catiônica de alto peso molecular que desestabiliza as partículas contidas na água desempenhando a função de coagulante natural (Ndabigengesere $e t$ al., 1995). Segundo Ndabigengesere e Narasiah (1998), as sementes de Moringa são uma alternativa viável de agente coagulante em substituição aos sais de alumínio, utilizados no tratamento de água em todo o mundo.

Diversos métodos para melhorar a eficiência da remoção de turbidez e cor têm sido propostos por pesquisadores, tais como a utilização da Moringa em pó (Amagloh e Benang, 2009; Mangale et al., 2012), preparação da solução por extração aquosa (Pritchard et al., 2010; Pise e Halkude, 2012) e preparação da solução por extração salina (Okuda et al., 2001; Madrona et al., 2012; Sánchez-Martín et al., 2012). Mesmo diante de diversos estudos, ainda não há padronização para tais procedimentos.

Assim, este trabalho tem como objetivo estabelecer a melhor dosagem de coagulante a ser utilizado e avaliar os diferentes tempos de sedimentação após a coagulação/floculação a fim de se otimizar o processo de tratamento de água superficial.

\section{MATERIAL E MÉTODOS}

A parte experimental deste trabalho foi realizada no Laboratório de Gestão, Controle e Preservação Ambiental, do Departamento de Engenharia Química - DEQ, da Universidade Estadual de Maringá - UEM. A água bruta utilizada nos ensaios, proveniente da bacia do Rio Pirapó foi coletada na Companhia de Saneamento do Paraná - Sanepar, localizada na cidade de Maringá - Paraná e as sementes de Moringa foram doadas pela Universidade Federal do Sergipe - SE.

\subsection{Obtenção do Coagulante}

O coagulante utilizado nos ensaios foi obtido da semente de Moringa oleifera Lam desengordurada segundo Sánchez-Martín et al. (2010) seguida de extração com solução salina $(\mathrm{NaCl})$. Para o preparo do coagulante salino de Moringa oleifera Lam., utilizou-se $1000 \mathrm{mg}$ de semente de Moringa desengordurada para 0,1L de água destilada, ou seja uma concentração de 


\section{9 a 22 de outubro de 2014 \\ Florianópolis/SC}

$1 \% \mathrm{~m} / \mathrm{v}$. A extração foi realizada por turbólise em liquidificador por 3 minutos com solução de $\mathrm{NaCl} 1 \mathrm{M}$ seguida de agitação em agitador magnético por 30 minutos (Madrona et al., 2010), após ser agitada, a solução passou por filtração em papel filtro qualitativo. A solução coagulante de Moringa foi preparada no momento do ensaio, pois estudos demonstram que o armazenamento da mesma por alguns dias pode ocasionar ineficiência do processo (Cardoso et al., 2008). O teor de proteínas presente no coagulante foi determinado segundo Lowry et al. (1951).

\subsection{Coagulação/Floculação da água superficial}

Os ensaios de coagulação/floculação foram realizados em Jar Test simples, Milan - Modelo JT 101/6 de seis provas, com regulador de rotação das hastes misturadoras utilizando-se béqueres contendo $400 \mathrm{~mL}$ de água bruta. Primeiramente foram realizados ensaios para determinar a melhor dosagem do coagulante Moringa testando as dosagens de 3,5; 7,0; 14,0; 21,0; 34,0; 48,0; 55,0; 75,0 mg/L de proteína. As condições operacionais do Jar Test foram de tempo de mistura rápida de 3 minutos com gradiente de mistura rápida de $100 \mathrm{rpm}$ e tempo de mistura lenta de 15 minutos com gradiente de mistura lenta de $15 \mathrm{rpm}$, após o ensaio de coagulação/floculação, o Jar Test foi desligado e as amostras mantidas em repouso por 60 minutos para que ocorresse a decantação do material floculado (Madrona et al., 2012). Em seguida foram coletados aproximadamente $30 \mathrm{~mL}$ de amostra de cada béquer para análise dos parâmetros de cor, turbidez e compostos com absorção em $\mathrm{UV}_{254 \mathrm{~nm}}$ a fim de verificar a eficiência de remoção pela comparação dos resultados com a água bruta.

Após a determinação da melhor dosagem repetiu-se o ensaio em Jar Test a fỉm de verificar qual o melhor tempo de sedimentação a ser utilizado, para isto as condições operacionais do equipamento foram as mesmas diferindo apenas nos tempos de sedimentação. Desta forma, ao final do ensaio de coagulação/floculação, o Jar Test foi desligado e as amostras mantidas em repouso para que ocorresse a decantação do material floculado. Em seguida foram coletados aproximadamente $30 \mathrm{~mL}$ de amostra de cada béquer, em intervalos de tempos de 15 minutos perfazendo um total de 60 minutos de decantação, para análise dos parâmetros de cor, turbidez e compostos com absorção em $\mathrm{UV}_{254 \mathrm{~nm}}$. Os parâmetros de cor e compostos com absorção em $\mathrm{UV}_{254 \mathrm{~nm}}$ foram avaliados por meio de análise realizada em espectrofotômetro DR $5000 \mathrm{Hach}$, a turbidez com o auxílio do turbidímetro 2100P Hach e pH no phmetro Thermo Scientific segundo procedimento recomendado pelo Standard Methods (Apha, 1995).

\section{RESULTADOS E DISCUSSÃO}

As características da água bruta utilizada nos ensaios de coagulação/floculação utilizando a Moringa como coagulante são apresentadas na Tabela 1. 
Tabela 1 - Características da água bruta utilizada nos ensaios de Jar Test

\begin{tabular}{ccc}
\hline Parâmetro & Unidade & Valores \\
\hline Cor aparente & $\mathrm{uH}^{(1)}$ & 339,50 \\
Turbidez & $\mathrm{NTU}^{(2)}$ & 75,00 \\
$\mathrm{UV}_{254 \mathrm{~nm}}$ & $\mathrm{~cm}^{-1}$ & 0,249 \\
$\mathrm{pH}$ & - & 7,98 \\
\hline
\end{tabular}

(1) unidade Hanzen $=$ mgPtCo L ${ }^{-1}$; (2) Unidades Nefelométricas de Turbidez (NTU).

Foram realizados ensaios com diferentes concentrações de coagulante salino variando de 3,5 à 75,0 mg/L de proteína conforme descrito anteriormente na metodologia com o objetivo de se encontrar a melhor dosagem de coagulante a ser utilizado. A Figura 1 apresenta as porcentagens de remoção de cor e turbidez obtidos para cada dosagem de coagulante em mg/L.

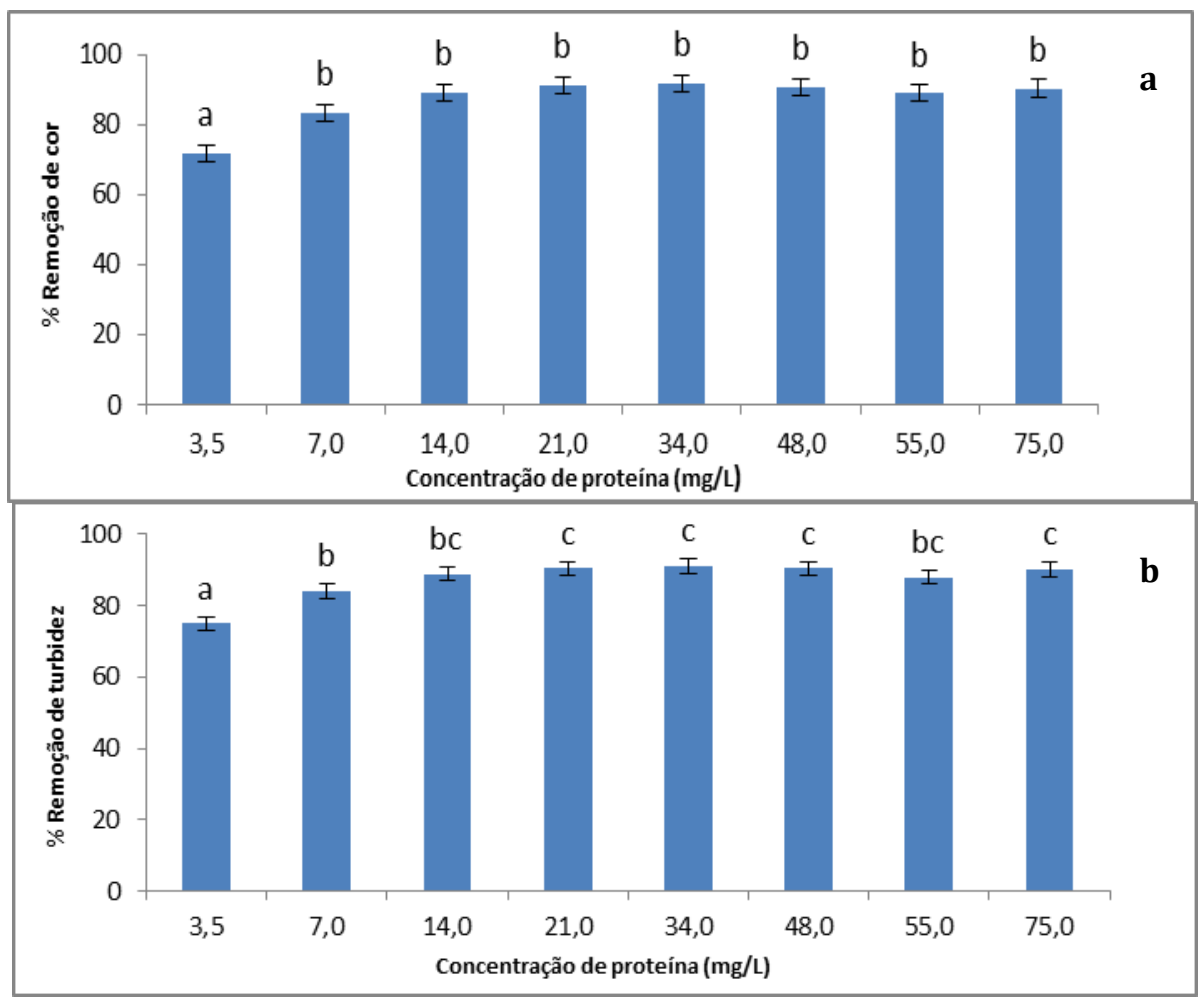

Figura 1 - Remoção de cor (a) e turbidez (b) para as diferentes concentrações de coagulante. *Os valores seguidos por mesma letra não diferem estatisticamente entre si, pelo teste de Tukey a 5\% de nível de significância.

No que diz respeito à remoção de cor, Figura 1 (a), pode-se verificar que não houve diferença estatística na remoção deste parâmetro nas dosagens de 7,0 à 75,0 mg/L de proteína coagulante. Já para a remoção de turbidez não foi observado diferença estatística nas dosagens de 
14,0 à 75,0 mg/L de coagulante, Figura 1 (b). Desta forma, no intuito de se utilizar menor volume do coagulante de Moringa na água a ser tratada e ao mesmo tempo manter a eficiência de coagulação, a melhor dosagem de coagulante seria de $14,0 \mathrm{mg} / \mathrm{L}$. Tanto o parâmetro de turbidez quanto o de cor obtiveram porcentagem de remoções similares para a dosagem de 14,0 mg/L, sendo $88,75 \%$ e $89,15 \%$ respectivamente. Valores próximos ao obtido neste estudo foram relatados por Nkurunziza et al. (2009) que utilizaram extrato salino para tratamento de água de 50 NTU de turbidez encontrando percentual de remoção de turbidez e cor de $87,5 \%$ e $87,7 \%$ respectivamente. A Figura 2 apresenta as porcentagens de remoção de compostos com absorção em $\mathrm{UV}_{254 \mathrm{~nm}}$ obtidos para cada dosagem de coagulante em mg/L.

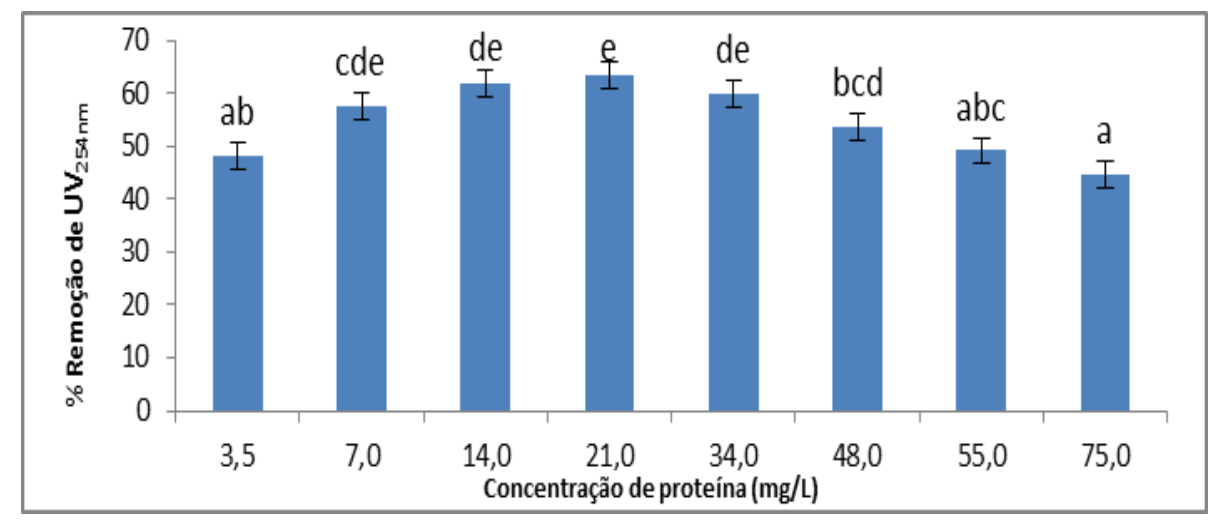

Figura 2 - Remoção de compostos com absorção em $U^{254 n m}$ para as diferentes concentrações de coagulante. *Os valores seguidos por mesma letra não diferem estatisticamente entre si, pelo teste de Tukey a 5\% de nível de significância.

Quanto ao percentual de remoção dos compostos com absorção em $\mathrm{UV}_{254 \mathrm{~nm}}$, expressos na Figura 2, este não apresentou diferença significativa no intervalo de dosagem de 14,0 à 34,0, havendo diminuição de remoção em concentrações de coagulante acima destas. Esta diminuição pode ser explicada pelo fato da moringa ser constituída de matéria orgânica e em concentrações elevadas pode acarretar em aumento na turbidez e na cor da água a ser tratada. Se comparado à turbidez e a cor, o parâmetro $\mathrm{UV}_{254 \mathrm{~nm}}$ apresentou menor remoção atingindo $62,0 \%$ na dosagem de $14,0 \mathrm{mg} / \mathrm{L}$.

Após definir a melhor dosagem de coagulante em 14,0 mg/L, repetiu-se os ensaios de coagulação/floculação na dosagem otimizada variando apenas os tempos de sedimentação que são expressos na Figura 3. 


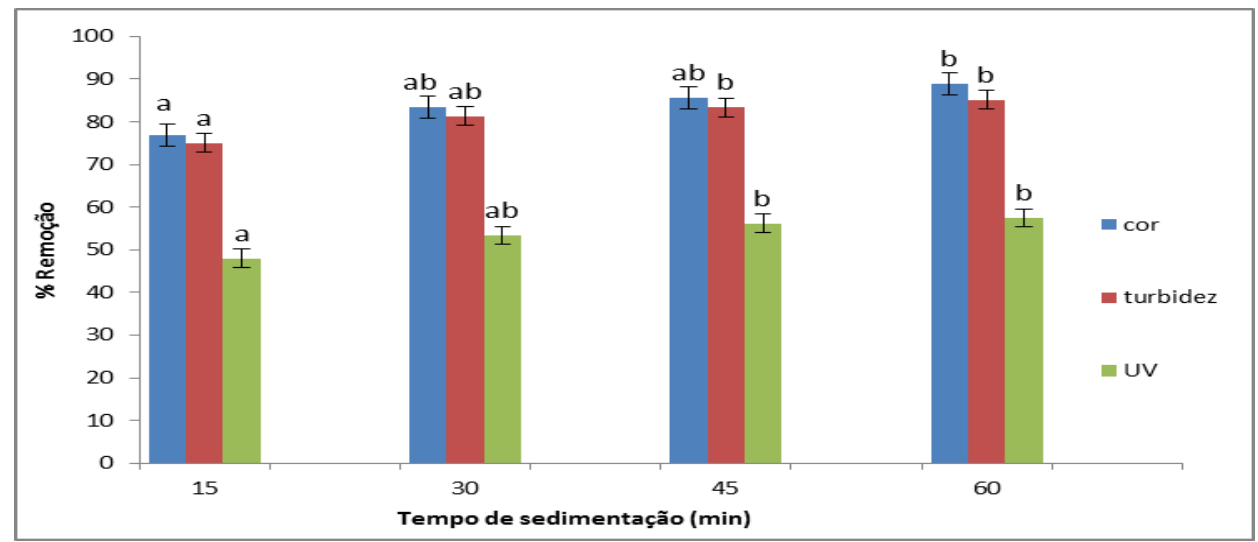

Figura 3 - Remoção de cor, turbidez e compostos com absorção em $U V_{254 n m}$ para os diferentes tempos de sedimentação. *Os valores seguidos por mesma letra não diferem estatisticamente entre si, pelo teste de Tukey a 5\% de nível de significância.

Na Figura 3 pode ser observado que de uma forma geral quanto maior o tempo de decantação, maior o valor obtido para a remoção dos parâmetros avaliados, desta forma o tempo de 15 minutos apresentou os resultados menos expressivos. Este comportamento se deve ao fato de que quanto maior o tempo de repouso, maior será a quantidade de partículas floculadas que serão decantadas. Porém ao analisar estatisticamente os resultados obtidos para os tempos de decantação de 30, 45 e 60 minutos não foi encontrada diferença significativa entre eles. Estudos envolvendo o uso de Moringa como coagulante para tratamento de águas superficiais utilizam tempo de sedimentação de 60 minutos (Madrona et al., 2012; Madrona et al., 2010) o que torna difícil a aplicação da Moringa no tratamento de água em larga escala já que em estações de tratamento de água o tempo de sedimentação utilizado é de 30 minutos. Desta forma, devido à ausência de diferença estatística entre os tempos de sedimentação de 30, 45 e 60 minutos e visando obter um processo de tratamento de água mais rápido sem existência de grandes comprometimentos na remoção dos parâmetros ao final do processo, escolheu-se como ideal o tempo de decantação de 30 minutos.

Os valores residuais e respectivas porcentagens de remoção dos parâmetros nas melhores condições, ou seja, tempo de sedimentação de 30 minutos e dosagem de proteína de 14,0 mg/L são expressos na Tabela 2.

Tabela 2 - Valor residual e porcentagem de remoção nas melhores condições.

\begin{tabular}{ccc}
\hline Parâmetros & Valores residuais & \% Remoção \\
\hline Cor & $56,02 \mathrm{uH}$ & 83,50 \\
Turbidez & $14,00 \mathrm{NTU}$ & 81,33 \\
$\mathrm{UV}_{254 \mathrm{~nm}}$ & $0,116 \mathrm{~cm}^{-1}$ & 53,41 \\
\hline
\end{tabular}


Observando a Tabela 2 pode-se perceber que as condições escolhidas de dosagem de proteína e tempo de sedimentação proporcionaram bons percentuais de remoção no processo de coagulação/floculação dos parâmetros de cor, turbidez e UV $\mathrm{UV}_{254 \mathrm{~nm}}$.

\section{CONCLUSÃO}

Com base no estudo desenvolvido foi possível constatar que tanto a concentração de coagulante de Moringa utilizada quanto o tempo de sedimentação adotado no processo de coagulação/floculação, ambos possuem influência na remoção de cor, turbidez e compostos com absorção em $\mathrm{UV}_{254 \mathrm{~nm}}$. Os melhores resultados obtidos foram para dosagem de coagulante de 14,0 mg/L e tempo de sedimentação de 30 minutos. Desta forma a Moringa oleifera Lam apresentouse eficiente na remoção de cor e turbidez, sendo desta maneira avaliada como um processo promissor na etapa de coagulação/floculação no tratamento de água. A utilização dessa semente pode ser considerada como um tratamento alternativo ao tratamento convencional.

\section{AGRADECIMENTOS}

Os autores agradecem ao apoio financeiro recebido pelo Conselho Nacional de Desenvolvimento Científico e Tecnológico (CNPQ), Coordenação de Aperfeiçoamento de Pessoal de Nível Superior (CAPES) e a Fundação Araucária.

\section{REFERÊNCIAS}

AMAGLOH, F. K.; BENANG, A. Effectiveness of Moringa oleifera seed as coagulant for water purification. Afr. J. Agric. Res, v.4, n.1, p. 119-123, 2009

APHA-American Public Health Association. Standard methods for the examination for water and wastewater. 19th ed. Washington, D.C.: APHA, 1995.

CARDOSO, K. C; BERGAMASCO, R.; COSSICH, E. S.; MORAES, C. K. Otimização dos tempos de mistura e decantação no processo de coagulação/floculação da água bruta por meio da Moringa oleífera Lam. Rev. Acta Sci. Tech., v. 30, n. 2, p. 193-198, 2008.

GALLÃO, M. I.; DAMASCENO, L. F.; BRITO, E. S. Chemical and structural evaluation of moringa seeds. Rev. Ciênc.Agron., v. 37, n.1, p. 106-109, 2006.

KAWAMURA, S. Effectiveness of natural polyelectrolytes in water treatment. J. Am. Water Work Assoc., v.79, n. 6, p. 88-91, 1991.

LOWRY, O. H., ROSEBROUGH, N. J., FARR, A. L.; RANDALL, R. J., J. Biol. Chem., v.193, p. $265,1951$. 


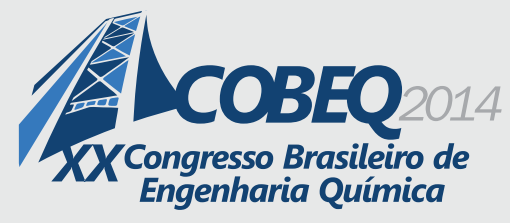

19 a 22 de outubro de 2014
Florianópolis/SC

MADRONA, G. S.; BRANCO, I. G.; SEOLIN, V. J.; ALVES FILHO, B. de A.; FAGUNDESKLEN, M. R.; BERGAMASCO, R. Evaluation of extracts of Moringa oleifera Lam seeds obtained with $\mathrm{NaCl}$ and their effects on water treatment. Acta. Sci. Technol., v. 34, n 3, p. 289293, 2012.

MADRONA, G. S.; SERPELlONI, G. B.; VIEIRA, A. M. S.; NISHI, L.; CARDOSO, K. C; BERGAMASCO, R. Study of the effect of saline solution on the extraction of the Moringa oleifera seed's active component for water treatment. Water Air Soil Poll, v. 211, p. 409-415, 2010.

MANGALE, S. M.; CHONDE, S. G.; JADHAV, A. S.; RAUT, P. D. Study of Moringa oleífera (drumstick) seed as natural absorbent and antimicrobial agent for river water treatment. IJNPR, v.2, n.1, p. 89-100, 2012.

NDABIGENGESERE, A., NARASIAH, K. S., Quality of Water Treated by Coagulation Using Moringa oleifera Seeds, Water Res., v. 32, n. 3, p. 781- 791, 1998.

NDABIGENGESERE, A.; NARASIAH, K. S.; TALBOT, B. G. Active agents and mechanism of coagulation of turbid waters using Moringa oleífera. Water Res., v. 29, n. 2, p. 703-710, 1995.

NISHI, L.; MADRONA, G. S.; GUILHERME, A. L. F.; VIEIRA, A. M. S.; ARAÚJO, A. A.; UGRI, M. C. B. A.; BERGAMASCO, R. Cyanobacteria removal by coagulation/floculation with seeds of the natural coagulant Moringa oleifera Lam. Chem. Eng. Transactions, v. 24, p. 11291134, 2011.

NKURUNZIZA, T.; NDUWAYEZU, J. B.; BANADDA, E. N.; NHAPI, I. The effect of turbidity levels and Moringa oleifera concentration on the effectiveness of coagulation in water treatment.Water Sci. Technol., v. 59, n. 8, p. 1551-1558, 2009.

OKUDA, T.; BAES, A. U.; NISHIJIMA, W.; OKADA, M. Isolation and characterization of coagulant extracted from Moringa oleifera seed by salt solution. Water Res., v. 35, n. 2, p. 405410, 2001.

PISE, C. P.; HALKUDE, D. S. A. Blend of natural and chemical coagulant for removal of turbidity in water. IJCIET, v. 3, n. 2, p. 188-197, 2012.

PRITCHARD, M.; CRAVEN, T.; MKANDAWIRE, T.; EDMONDSON, A. S.; O’NEILL, J. G. A comparison between Moringa oleifera and chemical coagulants in the purification of drinking water - An alternative sustainable solution for developing countries. Phys. Chem. Earth, v. 35, p. 798-805, 2010

SÁNCHEZ-MARTÍN, J.; BELTRÁN-HEREDIA, J.; PERES, J. A. Improvement of the flocculation process in water treatment by using Moringa oleifera seeds extract. Braz. J. Chem. Eng., v. 29, n. 3, p. 495-502, 2012.

SÁNCHEZ-MARTÍN, J.; GHEBREMICHAEL, K.; BELTRÁN-HEREDIA, J. "Comparison of single-step and two-step purified coagulants from Moringa oleifera seed for turbidity and DOC removal". Bioresour. Technol., v. 101, p. 6259-6261, 2010.

VIEIRA, A. M. S.; VIEIRA, M. F.; SILVA, G. F.; ARAÚJO, A. A.; FAGUNDES-KLEN, M. R.; VEIT, M. T.; BERGAMASCO, R. Use of Moringa oleifera Seed as a Natural Adsorbent for Wastewater Treatment. Water Air Soil Poll, v. 206, p. 273-281, 2010. 
19 a 22 de outubro de 2014

Florianópolis/SC 